\title{
Investigation of interface magnetic moment of Fe/Ge multilayer: A neutron reflectivity study
}

\author{
Surendra Singh ${ }^{\text {a) }}$ and Saibal Basu \\ Solid State Physics Division, Bhabha Atomic Research Center, Trombay, Mumbai 400 085, India \\ Mukul Gupta ${ }^{\text {b) }}$ \\ Laboratory of Neutron Scattering, ETHZ \& PSI, Paul Scherrer Institute, Villigen, Ch-5232, Switzerland \\ Mahesh Vedpathakz ${ }^{\mathrm{c})}$ and R. H. Kodama \\ Department of Physics, University of Illinois at Chicago, Chicago, Illinois 60607
}

(Received 13 July 2006; accepted 21 December 2006; published online 15 February 2007)

\begin{abstract}
$\mathrm{Fe} / \mathrm{Ge}$ multilayer sample was grown on $\mathrm{Si}(100)$ substrate by rf sputtering. X-ray diffraction shows that the Fe layers are polycrystalline whereas the Ge layers are amorphous in this sample. X-ray reflectometry and unpolarized neutron reflectometry techniques have been used to determine the structural parameters viz. individual layer thickness, interface roughness, and the density of the layers. Polarized neutron reflectometry has given magnetic moment depth profile for the multilayer thin film. There is a large reduction in magnetic moment for $\mathrm{Fe}$ atom on an average in the $\mathrm{Fe}$ layers. Magnetic moments of $\mathrm{Fe}$ at the interfaces are lower compared to the regions away from the interfaces. There is an asymmetry between magnetic moments at $\mathrm{Fe}$ on $\mathrm{Ge}$ interfaces and $\mathrm{Ge}$ on $\mathrm{Fe}$ interfaces as well. Superconducting quantum interference device measurement indicates that the sample is ferromagnetic at room temperature with a lower magnetic moment compared to bulk iron. (C) 2007 American Institute of Physics. [DOI: 10.1063/1.2450680]
\end{abstract}

\section{INTRODUCTION}

Metal-semiconductor interfaces display physical and chemical properties, ${ }^{1,2}$ which have stimulated many experimental and theoretical studies. ${ }^{3-6}$ Since the work of Datta $e t$ al. ${ }^{7}$ a new generation of electronic devices (spintronics), exploiting the spin degree of freedom of carriers in ferromagnet (FM)/semiconductor (SC) interfaces, ${ }^{8}$ have been envisaged, viz., spin field effect transistors, spin transistors, spin memories, etc. ${ }^{9}$ Injection of spin-polarized electrons from FM to SC presents a well-known problem of "conductivity mismatch" between the FM and the SC. ${ }^{10}$ It has been shown that this problem can be overcome by using a spin-dependent interface resistance between the FM and the SC. ${ }^{11,12}$ The property of interest, i.e., transport of spins across the FM/SC interface $^{6}$ will strongly depend on interface properties viz. roughness, alloying at interfaces, and magnetic moment at the $\mathrm{Fe} / \mathrm{Ge}$ interface. Some earlier efforts were focused on the issue relating to alloying, ${ }^{13}$ and diffusion of $\mathrm{Ge}$ and $\mathrm{Fe}$ at the interfaces, which affect the magnetic properties ${ }^{14,15}$ of $\mathrm{Fe}$ at $\mathrm{Fe} / \mathrm{Ge}$ interfaces. Recently, x-ray magnetic circular dichroism and resonant magnetic scattering experiments have shown evidence of very small induced magnetic moments on $\mathrm{Ge}\left(\sim 0.01 \mu_{B}\right)$ as a result of weak hybridization at the $\mathrm{Fe} / \mathrm{Ge}$ interface ${ }^{16}$ in $\mathrm{Fe} / \mathrm{Ge}$ multilayer sample grown by sputtering on Si [100] substrate. Magnetic moment at the FM/SC interface is one of the fundamental quantities required to understand spin-dependent transport properties in such systems.

\footnotetext{
${ }^{a)}$ Electronic address: surendra@barc.gov.in

${ }^{b)}$ Present address: UGC-DAE Consortium for Scientific Research, Mumbai, India.

${ }^{c}$ Present address: Vidya Pratishthan's Arts, Science and Commerce College, Baramati 413133, India.
}

The present work focuses on determination of magnetic moment densities at the interfaces of a Fe/Ge multilayer sample deposited by the sputtering technique.

The lattice constant of Ge is about twice that of bcc Fe with only a $1.3 \%$ lattice mismatch. It has been reported that for $\mathrm{Fe}$ grown on crystalline $\mathrm{Ge}$ at $150{ }^{\circ} \mathrm{C}$, a disordered interface region may cause magnetic dead layers with thicknesses of more than $10 \mathrm{~nm}$ as a result of intermixing. ${ }^{17} \mathrm{Sev}-$ eral theoretical attempts with epitaxial $\mathrm{Fe} / \mathrm{Ge}$ supercell structures to obtain $\mathrm{Fe}$ moment at $\mathrm{Fe} / \mathrm{Ge}$ interfaces ${ }^{18-21}$ also indicate that $\mathrm{Fe}$ moment reduces at the interfaces. Spin resolved electronic band structure calculations ${ }^{18-20}$ show that reduced atomic coordination, modified volume per atom, and the interface-induced electronic states strongly affect the magnetic moment of iron at $\mathrm{Fe} / \mathrm{Ge}$ interface. Interface magnetic moments are also strongly dependent on the crystalline state of $\mathrm{Fe}$ and $\mathrm{Ge}$ as well as the deposition sequence. X-ray photoelectron spectroscopy (XPS) showed that when crystalline $\mathrm{Fe}$ is deposited on amorphous $\mathrm{Ge}$, the semiconductor penetrates deeper inside the iron layer compared to the situation when the amorphous semiconductor is deposited on $\mathrm{Fe}^{22}$ Recently, Zhou et al. ${ }^{23}$ have performed first-principles electronic structure calculations to investigate the structural and magnetic properties of bcc $\mathrm{Fe}$ on $\mathrm{Ge}(001)$ system with up to three Fe layers. The results of their calculation for the crystalline bcc $\mathrm{Fe}$ on crystalline Ge substrate gives an enhancement of $\mathrm{Fe}$ moment at the interface. With this background, magnetic moment at the interfaces of $\mathrm{Fe} / \mathrm{Ge}$ multilayers depends on cumulative effect of microscopic structure, alloying and roughness present at the interfaces. X-ray diffraction (XRD) measurement of the present sample shows that the Ge layers are amorphous, while the Fe layers are in polycrystalline state. 
Earlier we had undertaken polarized neutron reflectometry (PNR) and superconducting quantum interference device (SQUID) measurement of a $\mathrm{Fe} / \mathrm{Ge}$ multilayer thin film ${ }^{24}$ with an aim to obtain the depth profile of its magnetic moment as well as macroscopic magnetic structure of the film. We could demonstrate conclusively that there was a large reduction in the magnetic moment of $\mathrm{Fe}$ in the multilayer on an average. Due to lower thickness of the $\mathrm{Fe}$ layers ( $\sim 6$ monolayers) and limited momentum transfer range in that experiment it was not possible to specifically target the interface magnetic moment in the previous experiment. The present sample has ten bilayers of $\mathrm{Fe}$ and $\mathrm{Ge}$, of nominal thickness 75 and $55 \AA$ respectively, sputter deposited on a Ge buffer layer. In this work, from PNR experiment, we have clearly demonstrated that there is a large reduction of Fe moment in the sample and the Fe moment at the interfaces are substantially lower compared to regions away from the interfaces. Also we find that the magnetic moment of $\mathrm{Fe}$ at the interfaces depend on the deposition sequence, i.e., $\mathrm{Fe}$ on $\mathrm{Ge}$ has different moment compared to $\mathrm{Ge}$ on $\mathrm{Fe}$ interface. We attribute this asymmetry to asymmetric diffusion at the two interfaces observed by Marika et al. ${ }^{22}$ in their XPS experiment.

\section{EXPERIMENT}

The Fe/Ge multilayer sample used in the present study was grown by rf sputtering technique on $\mathrm{Si}(100)$ substrate at base pressure of $5 \times 10^{-8}$ Torr and at deposition pressure of $4 \times 10^{-3}$ Torr. The Si (100) substrate was first cleaned for both organic and inorganic contaminations before the film was deposited. During the growth of the film the substrate was kept at an elevated temperature of $250{ }^{\circ} \mathrm{C}$. First a Ge buffer layer of thickness about $100 \AA$ was grown on the substrate. Ten bilayers of $\mathrm{Fe}$ and $\mathrm{Ge}$, of nominal thickness 75 and $55 \AA$, respectively, were deposited on the buffer layer. A thin layer of $30 \AA$ of Au was deposited as a protective capping layer at the air-film interface. The designed structure of the multilayer sample can be represented as: $\mathrm{Si}(100) / \mathrm{Ge}_{100} \AA /\left[\mathrm{Fe}_{75} \AA / \mathrm{Ge}_{55} \AA\right] \times 10 / \mathrm{Au}_{30 \AA}$. The sample thickness was monitored by quartz crystal system during growth.

In neutron reflectometry (NR) [or x-ray reflectometry (XRR)], one observes specular reflectivity profile of neutrons as a function of angle of incidence (or momentum transfer) from the surface of the thin film. Layer thickness, density profile, and interface roughness can be obtained from reflectivity measurement. Magnetic moment density profile can be obtained from PNR measurements. Experimentally, incident neutrons with spins polarized parallel (spin up) and antiparallel (spin down) to the sample magnetization are reflected off the surface of the sample at grazing incidence. Details of the NR, PNR, and XRR can be obtained from Refs. 25-27. PNR is able to yield magnetic moment density in a multilayer system as a function of depth, with high $\operatorname{accuracy}^{25,27,28}$ and has an advantage over other bulk magnetic measurement techniques in this regard. The neutron reflectometry experiments at room temperature, both in polarized and unpolarized mode, were performed on AMOR

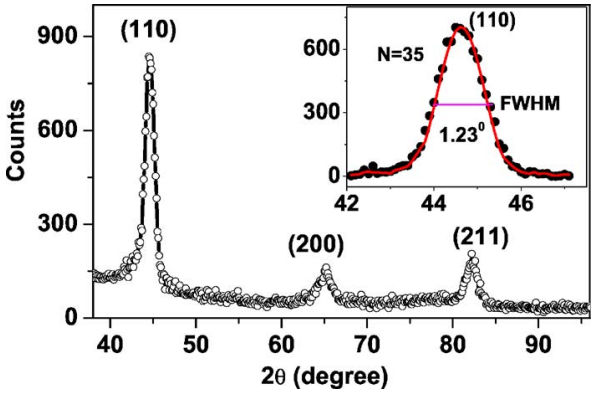

FIG. 1. X-ray diffraction pattern for the multilayer $\mathrm{Fe} / \mathrm{Ge}$ sample. The peaks correspond to bcc Fe. The inset of figure shows Fe (110) peak with a full width of half maximum equal to $1.23^{\circ}$. The open circles are the experimental data, while the line is fit to measurements using Eq. (2).

reflectometer at SINQ neutron source, PSI Switzerland. ${ }^{29}$ To align the magnetic moment in the sample, a saturation magnetic field was applied parallel to the surface of sample during polarized runs. The XRD and XRR measurements were obtained, using a $\mathrm{Cu} K \alpha$ source. The magnetic hysteresis loop of the multilayer sample was recorded on a quantum design MPMS5-SQUID magnetometer. The film was oriented with its plane parallel to the applied magnetic field. The magnetization curves were measured at 77 and $300 \mathrm{~K}$.

The neutron reflectometry data, both in polarized and unpolarized mode, have been analyzed using a genetic algorithm (GA) based $\chi^{2}$ minimization program, ${ }^{30}$ which uses a matrix method ${ }^{25}$ for generating the reflectivity pattern for a given set of physical parameters (i.e., layer thickness, density, interface roughness, and magnetic moment for PNR) of the system. The roughness parameters were the last variables to be fitted to "fine-tune" the fits. The interface roughness parameter extracted from NR is an effective roughness, which convolutes the effect of interdiffusion as well as true roughness. ${ }^{31,32}$ Roughness has the effect of reducing the specular intensity at any given momentum transfer value. The fitting program takes into account the reduction in specular intensity by introducing a multiplicative exponential factor, as proposed by Nevot and $\mathrm{Croce}^{32}$ and given by

$$
r_{j}^{m}=r_{j}^{0} e^{-4 q_{j} q_{j-1} \sigma_{j}^{2}},
$$

where $\sigma_{j}$ and $q_{j}$ are the root-mean-square roughness amplitude and the perpendicular wave vectors, respectively, at the $j$ th interface. $r_{j}^{0}$ is the Fresnel reflectivity for an ideal sample with perfectly smooth surface and interfaces, $r_{j}^{m}$ is the measured specular intensity. X-ray reflectometry data has been analyzed using the XOP software. ${ }^{33}$

\section{RESULTS AND DISCUSSION}

\section{A. Structural properties}

Figure 1 shows the XRD pattern of the sample. The measured peaks at $44.70^{\circ}, 65.18^{\circ}$, and $82.26^{\circ}$ correspond to [110], [200], and [211] peaks of bcc iron, respectively, with a cell parameter of $2.860 \AA \AA^{34}$ The absence of any Ge peak in the XRD pattern indicates that the Ge layers are amorphous in this film. This result is in agreement with the earlier observation by Majkrzak et al. ${ }^{35}$ The broadening of the XRD peaks for Fe can be attributed to the finite thickness of the 


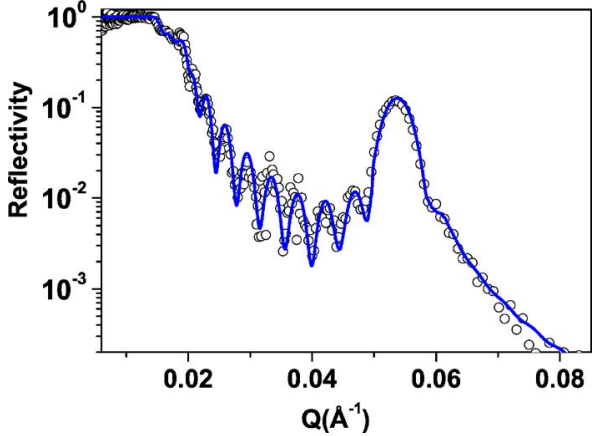

FIG. 2. The unpolarized neutron reflectivity data for the sample. The open circles are the experimental data and the solid line is the best fit to the data.

individual Fe layers. The crystallite size can be estimated from the full width at half maximum (FWHM) of the $\mathrm{Fe}$ [110] diffraction peak which is equivalent to the Fe layer thickness in the present case, due to strong [110] texture along the growth direction in the film. The inset of Fig. 1 shows the measured intensities of Fe [110] peak (solid circles) along with the fit (solid line), using the following function:

$$
\operatorname{I\alpha I}_{0}\left|\frac{\sin \left(\frac{N Q d}{2}\right)}{\sin \left(\frac{Q d}{2}\right)}\right|^{2},
$$

where $N, Q$, and $d$ are the number of $\mathrm{Fe}(110)$ planes, momentum transfer, and spacing of planes, respectively. The value of $N$ from the fit is 35. For $d$ value of $2.02 \AA$ for [110] plane, it gives the thickness of Fe layer equal to $70.7 \AA$. We observe that this value is close to the values obtained from XRR and NR.

The layer thicknesses for the Fe and Ge layers in the film and their densities were determined from unpolarized NR measurements as well as from XRR. Figure 2 shows the experimental data (circles) for NR along with the best fit (continuous line). The extracted parameters from the fit (i.e., layer thicknesses and the corresponding densities) have been shown in Table I. The densities of the Fe and Ge layers are about $93 \%$ of their bulk values. This suggests growth of the layers with small porosity. The layer thicknesses of $\mathrm{Fe}$ and Ge layers, obtained from the fit, are $75 \AA( \pm 6 \AA)$ and $51 \AA( \pm 4 \AA)$, respectively. Since the NR data was taken over a narrower $Q$ range, we also obtained XRR data from the film over a larger $Q$ range, extending up to $0.4 \AA^{-1}$. We compared the fitted parameters from XRR with NR results.

TABLE I. The parameters of the Fe/Ge multilayer as obtained by NR.

\begin{tabular}{cccc}
\hline \hline & \multicolumn{3}{c}{ Unpolarized neutron reflectivity } \\
\cline { 2 - 4 } Structure material & $\begin{array}{c}\text { Thickness } \\
(\AA)\end{array}$ & $\begin{array}{c}\text { Density } \\
\left(\mathrm{g} / \mathrm{cm}^{3}\right)\end{array}$ & $\begin{array}{c}\text { Roughness } \\
(\AA)\end{array}$ \\
\hline $\mathrm{Au}$ & $27 \pm 3$ & $18.0 \pm 1.20$ & $7.0 \pm 2.4$ \\
$\mathrm{Ge}$ & $51 \pm 4$ & $4.90 \pm 0.35$ & $10.0 \pm 2.5$ \\
$\mathrm{Fe}$ & $76 \pm 6$ & $7.40 \pm 0.40$ & $12.0 \pm 3.0$ \\
$\mathrm{Ge}($ buffer $)$ & $78 \pm 6$ & $5.0 \pm 0.30$ & $8.5 \pm 3.0$ \\
$\mathrm{Si}(100)$ & - & $2.1 \pm 0.20$ & $7.0 \pm 2.0$ \\
\hline \hline
\end{tabular}

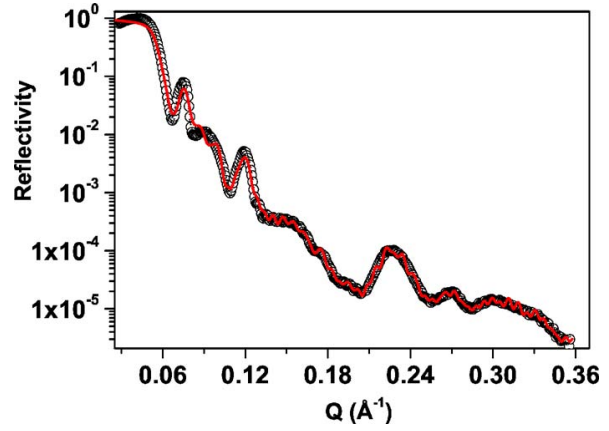

FIG. 3. The X-ray reflectivity data from the sample (open circles). The solid line shows the fit to XRR measurement.

Fit to the XRR gives layer thicknesses for the Fe and $\mathrm{Ge}$ layers 68 and $53 \AA$, respectively, which lie within the experimental error bars in the NR results. Figure 3 shows the experimental XRR data (solid circles) along with the fitted profile (continuous line). The Bragg peak in NR at $Q$ $=0.05 \AA^{-1}$, corresponds to bilayer thickness of $126 \AA$ in real space $(d=2 \pi / Q)$. We could observe Bragg peaks up to third order in XRR, which confirms that the interlayer roughness in the film is small. The interlayer roughness obtained from the fits to NR and XRR are in the range of 8 to $12 \AA$ for the film. The close match between the NR and XRR results confirms the accuracy of layer thicknesses of the thin film sample, obtained from NR. These parameters were used unaltered for obtaining magnetic depth profile from our PNR data.

\section{B. Magnetic properties}

For obtaining magnetic moment densities in Fe layers of the sample, PNR profiles were obtained from the sample for spin-up (+) and spin-down (-) neutrons with respect to the sample polarization in the plane of the thin film. An in-plane field of $400 \mathrm{G}$ was applied on the sample, which was enough to saturate its in-plane magnetization, confirmed from SQUID experiments, presented later. Figure 4 shows the results of PNR measurements. The closed and open circles in Fig. 4 shows the reflectivity data for incident neutron with spin up (solid circle) and spin down (open circle), respec-

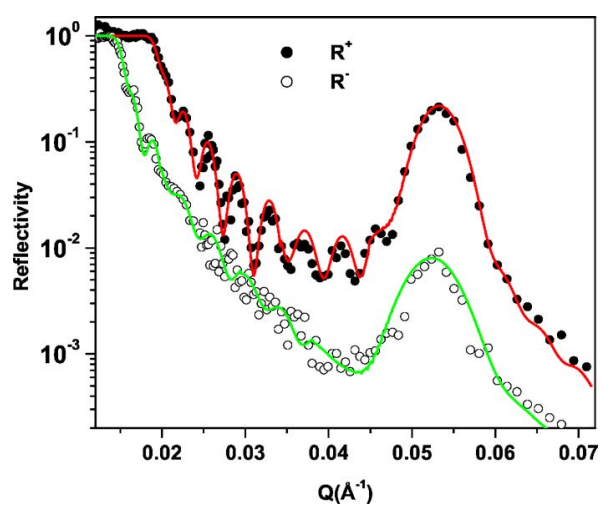

FIG. 4. Polarized neutron reflectivity plot for $\mathrm{Fe} / \mathrm{Ge}$ multilayer sample. Closed and open circles are the experimental reflectivity data for spin-up $\left(R^{+}\right)$and spin-down $\left(R^{-}\right)$neutrons, respectively. The solid lines are the best fits. 


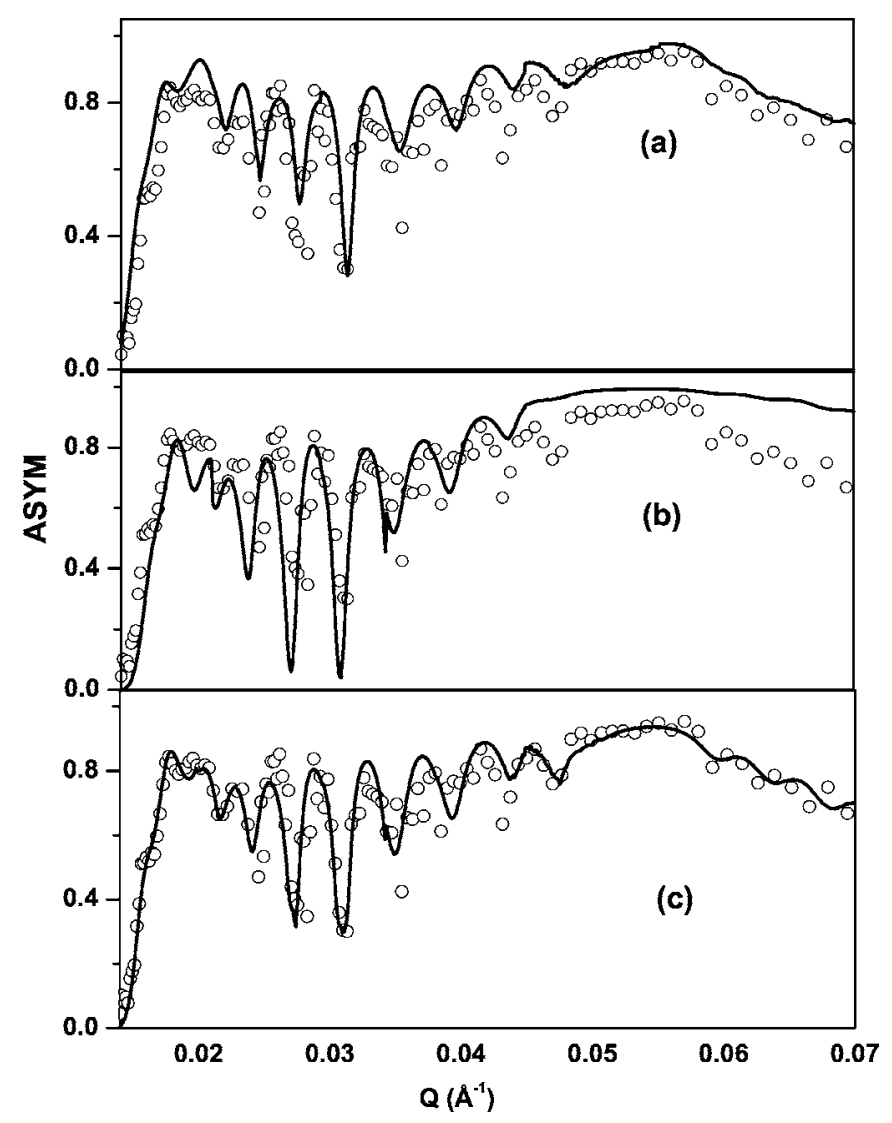

FIG. 5. The spin asymmetry, defined in Eq. (3), is plotted against $Q$. Open circles are the experimental data and solid lines are obtained from best fit for spin-up and spin-down neutrons, for three different models as shown in Figs. 6(a)-6(c)

tively. The solid lines are the best fits to the measurements $R^{+}(Q)$ and $R^{-}(Q)$ for the model of magnetic moment density profile described below. Instead of looking for best fits for the spin-up and spin-down data individually, we have looked for the magnetic moment density profile that best fits the spin asymmetry (ASYM) parameter, defined in Eq. (3). This parameter is less sensitive to interface roughness $(\sigma)$ than the reflectivity profiles and models the alloying at the interfaces better. The ASYM parameter is given by

$$
\operatorname{ASYM}(Q)=\frac{R^{+}(Q)-R^{-}(Q)}{R^{+}(Q)+R^{-}(Q)}
$$

The experimental ASYM parameter is shown in Fig. 5 (open circles) along with best fits for three different models of magnetic depth profile (solid lines) in Figs. 5(a)-5(c). All the physical parameters obtained from unpolarized NR measurement (i.e., thickness, densities, and interface roughness) were kept fixed for analyzing the PNR data and only the depth profile of the magnetic moment density in Fe layers were varied. Using the physical density obtained from NR and magnetic moment density obtained from PNR, the magnetic moment per Fe atom was evaluated. We considered three models for the magnetic moment density in the Fe layer as shown schematically in Figs. 6(a)-6(c). Model (a) corresponds to a uniform magnetic moment density for the entire Fe layer, model (b) assumes a magnetically dead layer at the $\mathrm{Fe} / \mathrm{Ge}$ interfaces with a uniform density away from the in-

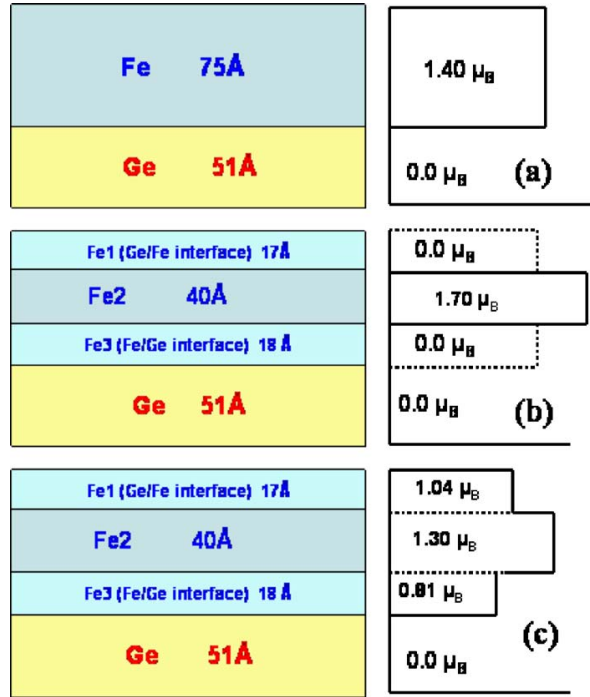

FIG. 6. Schematic of three different models, used for analyzing PNR data: (a) uniform magnetic moment density in Fe layer; (b) magnetically dead layers at interfaces; and (c) three Fe sublayers with different magnetic moments.

terfaces, and model (c) assumes lower Fe moments at the interfaces. Models (b) and (c) split each Fe layer into three sublayers, Fe1, Fe2, and Fe3. For models (b) and (c) we obtained the best fits by varying thicknesses of the sublayers, viz., $\mathrm{Fe} 1, \mathrm{Fe} 2, \mathrm{Fe} 3$, and the moments in the corresponding layers, while keeping the total layer thicknesses for $\mathrm{Fe}$ and Ge layers and their physical densities fixed at values obtained from NR and XRR. There is an overall reduction in Fe magnetic moment from its bulk value of $2.2 \mu_{B}$ in the multilayer sample. Best fits for the models (a)-(c) are shown in Figs. 5(a)-5(c) respectively, as solid lines. For model (a) we obtained a uniform magnetic moment of $1.40 \mu_{B}$ per Fe atom from the best fit. Model (b) gives $1.7 \mu_{B}$ for the central part of the Fe layers with about $20 \AA$ dead layers at the interfaces. The best fit to model (c) gives a magnetic moment of $1.30 \pm 0.08 \mu_{B}$ per Fe over $40 \AA$ at the center of the Fe layer (Fe2 sublayer) away from the interfaces, $0.81 \pm 0.04 \mu_{B}$ for $\mathrm{Fe} 3$ sublayer (Fe on $\mathrm{Ge}$ ) and $1.04 \pm 0.05 \mu_{B}$ for Fe1 sublayer $(\mathrm{Ge}$ on $\mathrm{Fe})$. Of the three models we got the best fit for model (c) over the entire $Q$ range as is evident from Fig. $5(c)$.This observation is central in the present set of experiments.

We believe that the inequality in the Fe moments at two interfaces ( $\mathrm{Fe} 1$ and $\mathrm{Fe} 3)$ is a clear signature of asymmetry of Ge diffusion in the Fe layer at the two interfaces for the case of amorphous Ge layers, conjectured from XPS earlier. ${ }^{22}$ According to this model the moment at $\mathrm{Fe} 3$ (Fe on $\mathrm{Ge}$ ) interface is expected to be less than that at $\mathrm{Fe} 1$ ( $\mathrm{Ge}$ on $\mathrm{Fe}$ ) because when polycrystalline $\mathrm{Fe}$ is deposited on amorphous $\mathrm{Ge}$, there is more interdiffusion than the other case, i.e., Ge deposition on Fe. The present result endorses this model. The sample was grown on $\mathrm{Si}$ substrate at $250{ }^{\circ} \mathrm{C}$ and there is possibility of forming alloy at the interface as it was shown ${ }^{15}$ that for temperatures above $160{ }^{\circ} \mathrm{C}$ a $\mathrm{Ge}-\mathrm{Fe}$ alloy exists. It had been conjectured earlier from electron energy loss spectroscopy that band hybridization in amorphous $\mathrm{Fe}_{x} \mathrm{Ge}_{1-x}$ alloys can greatly reduce magnetic moment ${ }^{36}$ near $x \sim 0.5$. The lower 


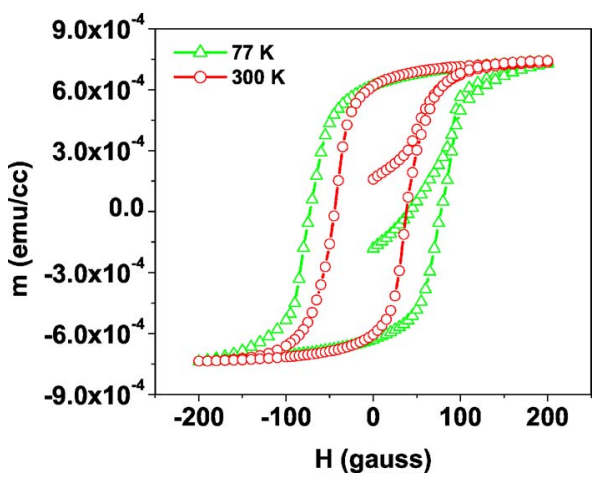

FIG. 7. The hysteresis loops of the sample measured at $77 \mathrm{~K}(\triangle)$ and $300 \mathrm{~K}$ $(\bigcirc)$ in a SQUID magnetometer. The $y$ axis is the total magnetic moment of the sample in electromagnetic unit.

magnetic moment for $\mathrm{Fe}$ at the interfaces in the present sample is attributed to the cumulative effect of roughness and interdiffusion leading to band hybridization. The present results favor model (c) [lower interface moment] compared to model (b) [magnetic dead layers at interfaces].

A comparison between the existing theoretical work and the present results is relevant at this point. Reduction of magnetic moments at the idealized interfaces had been predicted theoretically. ${ }^{18,19}$ But in such cases the magnetic moment relaxes back to the bulk value within two or three atomic layers. In the present case we find that Fe moment is low over thickness of about $20 \AA$ at the interfaces, which is much larger than theoretical predictions. We attribute this to the nonideality of the interfaces, related to roughness and diffusion. Further computational attempts with realistic interface geometries is necessary in this regard.

We have also determined the macroscopic magnetic phase of the multilayer sample at room temperature and at liquid $\mathrm{N}_{2}$ temperature by measuring the hysteresis loop on a SQUID magnetometer. For this measurement, a rectangular piece of sample of dimension (approximately) $3.0 \mathrm{~mm}$ $\times 4.0 \mathrm{~mm}$ was used. Figure 7 shows the magnetization hysteresis loop at temperatures 70 and $300 \mathrm{~K}$, respectively, for the sample. Apart from the small difference in the coercive field of the sample at two temperatures, which is clear from the figure, we observe ferromagnetic loops with relatively low saturation fields. The magnetic moment in the sample saturates at an applied field of about $100 \mathrm{G}$. The saturated magnetization of the sample was $7.4 \times 10^{-4} \mathrm{emu} / \mathrm{cm}^{3}$. We have estimated the magnetic moment of the $\mathrm{Fe}$ atom in this rectangular sample using the saturated magnetization measured by SQUID. Since SQUID magnetometry measures the total moment of the sample, high accuracy in determining the sample area (in this case $3 \mathrm{~mm} \times 4 \mathrm{~mm}$ ) is essential. Using the density and total thickness of the Fe layers $(\sim 750 \AA$, i.e., thickness of $10 \mathrm{Fe}$ layers), as obtained from the NR data, the magnetic moment obtained from SQUID corresponds to $1.17 \mu_{B}$ per Fe atom. This value is reasonably close to the magnetic moment we obtained from microscopic PNR experiments $\left(1.05 \mu_{B}\right)$. The coercive field at $70 \mathrm{~K}$ was more than twice as compared to that at room temperature. At high temperature (below critical temperature), thermal excitations release domain walls from their local energy minima and results in an increase of domain orientation distribution, which reduces coercive field.

\section{SUMMARY AND CONCLUSION}

The structure of a Fe/Ge multilayer sample and its microscopic and macroscopic magnetic properties has been characterized using XRD, XRR, NR, PNR, and SQUID measurements. Such semiconductor/ferromagnetic modulated structures are of interest regarding spin injection in a semiconductor material. The Fe layer has a bcc structure and the Ge layer is amorphous in this film. The average magnetic moment of Fe obtained from PNR measurement is substantially lower $\left(1.05 \mu_{B}\right)$ corresponding to bulk Fe magnetic moment of $2.2 \mu_{B}$. The PNR study shows a large reduction in magnetic moment of Fe atoms at the interfaces compared to regions away from the interfaces. The SQUID hysteresis loop has shown that the sample is a ferromagnet at room temperature with a low saturation field of about $100 \mathrm{G}$.

\section{ACKNOWLEDGMENTS}

The authors would like to thank G. Ravikumar and Y. Goud, Technical Physics and Prototype Engineering Division, Bhabha Atomic Research Centre, Mumbai, India for SQUID measurements.

${ }^{1}$ L. J. Brillson, Surf. Sci. Rep. 2, 123 (1982).

${ }^{2}$ G. Lelay, Surf. Sci. 132, 169 (1983).

${ }^{3}$ A. Perlov, V. Popescu, and H. Ebert, J. Appl. Phys. 91, 8798 (2002).

${ }^{4}$ R. R. Gareev, D. E. Burgler, R. Schreiber, H. Braak, M. Buchmeier, and P. A. Grunberg, Appl. Phys. Lett. 83, 1806 (2003).

${ }^{5}$ P. Walser, M. Schleberger, P. Fuchs, and M. Landolt, Phys. Rev. Lett. 80, 2217 (1998).

${ }^{6}$ S. A. Wolf, D. D. Awschalom, R. A. Buhrman, J. M. Daughton, S. von Molnár, M. L. Roukes, A. Y. Chtchelkanova, and D. M. Treger, Science 294, 1488 (2001).

${ }^{7}$ S. Datta and B. Das, Appl. Phys. Lett. 56, 665 (1990).

${ }^{8}$ I. Žutić, J. Fabian, and S. Das Sarma, Rev. Mod. Phys. 76, 323 (2004).

${ }^{9}$ M. E. Flatté and G. Vignale, Appl. Phys. Lett. 78, 1273 (2001); I. Žutić J.

Fabian, and S. Das Sarma, Phys. Rev. Lett. 88, 066603 (2002).

${ }^{10}$ G. Schmidt, D. Ferrand, L. W. Molenkamp, A. T. Filip, and B. J. van Wees, Phys. Rev. B 62, R4790 (2000).

${ }^{11}$ E. I. Rashba, Phys. Rev. B 62, R16267 (2000).

${ }^{12}$ A. Fert and H. Jaffrès, Phys. Rev. B 64, 184420 (2001).

${ }^{13}$ M. Schleberger, P. Walser, M. Hunziker, and M. Landolt, Phys. Rev. B 60, 14360 (1999).

${ }^{14}$ G. W. Anderson, P. Ma, and P. R. Norton, J. Appl. Phys. 79, 5641 (1996).

${ }^{15}$ P. Ma and P. R. Norton, Phys. Rev. B 56, 9881 (1997).

${ }^{16}$ J. W. Freeland, R. H. Kodama, M. Vedpathak, S. C. Erwin, D. J. Keavney, R. Winarski, P. Ryan, and R. A. Rosenberg, Phys. Rev. B 70, 033201 (2004).

${ }^{17}$ G. A. Prinz, in Ultrathin Magnetic Structures II: Measurement Techniques and Novel Magnetic Properties, edited by B. Heinrich and J. A. C. Bland (Springer, New York, 1994), p. 35.

${ }^{18}$ A. J. Freeman and C. L. Fu, J. Appl. Phys. 61, 3356 (1987).

${ }^{19}$ W. H. Butler, X. G. Zhang, X. Wang, J. van Ek, and J. M. MacLaren, J. Appl. Phys. 81, 5518 (1997).

${ }^{20}$ C. L. Fu, A. J. Freeman, and T. Oguclii, Phys. Rev. Lett. 54, 2700 (1985).

${ }^{21}$ I. Cabria, A. Ya Perlov, and H. Ebert, Phys. Rev. B 63, 104424 (2001).

${ }^{22}$ M. Schleberger, P. Walser, M. Hunziker, and M. Landolt, Phys. Rev. B 60, 14360 (1999).

${ }^{23}$ X. H. Zhou, X. S. Chen, L. Z. Sun, Y. L. Sun, and W. Lu, J. Phys. D 38, 1055 (2005).

${ }^{24}$ S. Singh, S. Basu, M. Vedpathak, R. H. Kodama, R. Chitra, and Y. Goud, Appl. Surf. Sci. 240, 251 (2005). 
${ }^{25}$ S. J. Blundell and J. A. C. Bland, Phys. Rev. B 46, 3391 (1992).

${ }^{26}$ Polarised Neutron Reflection, edited by J. A. C. Bland, Vol. I of Ultrathin Magnetic Structures (Springer-Verlag, Berlin, 1994), pp. 305-342.

${ }^{27}$ G. P. Felcher, K. E. Gray, R. T. Kampwirth, and M. B. Brodsky, Physica B 136, 59 (1986).

${ }^{28}$ H. Zabel, Physica B 198, 156 (1994).

${ }^{29}$ M. Gupta, T. Gutberlet, J. Stahn, P. Keller, and D. Clemens, Pramana, J. Phys. 63, 57 (2004).

${ }^{30}$ S. Singh and S. Basu, Solid State Phys. 44, 257 (2001).
${ }^{31}$ M. Wormington, Philos. Mag. Lett. 74, 211 (1996).

${ }^{32}$ L. Névot and P. Croce, Rev. Phys. Appl. 15, 761 (1980).

${ }^{33}$ The data analysis package for $\mathrm{x}$-ray reflectivity, XOP package is available at http://www.esrf.fr/computing/scientific/xop.

${ }^{34}$ E. A. Owen and G. I. Williams, J. Sci. Instrum. 31, 49 (1954).

${ }^{35}$ C. F. Majkrzak, J. D. Axe Peter and P. Boni, J. Appl. Phys. 57, 3657 (1985).

${ }^{36}$ T. I. Morrison, M. B. Brodsky, N. J. Zaluzec, and L. R. Sill, Phys. Rev. B 32, 3107 (1985) 